\title{
THE WORD PROBLEM FOR FREE LATTICE-ORDERED GROUPS (AND SOME OTHER FREE ALGEBRAS)
}

\author{
TREVOR EVANS
}

\begin{abstract}
It is known that in the lattice of all varieties of $l$-groups, there is a unique maximal proper subvariety. Based on this fact we give a simple algorithm for deciding the word problem in free $l$-groups. Some other applications are also given.
\end{abstract}

The word problem for free $l$-groups was shown to be solvable by Holland and McCleary [4] by techniques appropriate to l-groups. We give here a brief universal algebra proof of this theorem which is not much use in studying further properties of $l$-groups but which generalizes to any finitely presented variety $\mathbf{V}$ for which there is some effectively given list of identities, none of which are satisfied by $\mathbf{V}$ but at least one of which holds in any proper subvariety or $\mathbf{V}$. It was pointed out in Evans, Neff, and Mandelberg $[\mathbf{1}$, p. 261], that the word problem is solvable for free algebras in any finitely presented equationally complete variety and what we have here is a mild generalization of this result.

We recall that a lattice ordered group is a set $G$ which is (i) a lattice under a partial order $x \leq y$, and (ii) a group under the operations $x y, x^{-1}$, 1 , with the order relation compatible with the group multiplication, i.e. $x \leq y$ implies $a x \leq a y$, $x a \leq y a$. The class of all such algebras is a variety or equationally defined theory, as is easily seen if we express the lattice axioms as identities in terms of the join and meet, the group axioms as identities, and the compatibility properties by

$$
\begin{aligned}
& x(y \wedge z)=x y \wedge x z, \quad(y \wedge z) x=y x \wedge z x, \\
& x(y \vee z)=x y \vee x z, \quad(y \vee z) x=y x \vee z x .
\end{aligned}
$$

It is this class of equationally defined structures we have in mind when we talk about the variety of $l$-groups or the equational theory of $l$-groups.

THEOREM. The word problem for free l-groups is solvable.

PROOF. This decision problem is equivalent to that of deciding for any given identity $u\left(x_{1}, x_{2}, x_{3}, \ldots\right)=v\left(x_{1}, x_{2}, x_{3}, \ldots\right)$ in the theory of $l$-groups whether or not the identity is a consequence of the axioms and hence holds in all $l$-groups.

There is an algorithm $M_{1}$ which enumerates all identities, which hold in all $l$ groups and only such identities. If $u=v$ is an l-group identity which holds in all $l$-groups. it will eventually appear in this enumeration.

Let $\mathbf{L}$ be the variety of all $l$-groups. In the lattice of all subvarieties of $\mathbf{L}$, there is a largest proper subvariety $\mathbf{N}$ which is covered by $\mathbf{L}$ (Holland $[\mathbf{3}]$ ). $\mathbf{N}$ is the variety

Received by the editors July 2, 1985 and, in revised form, August 22, 1985.

1980 Mathematics Subject Classification (1985 Revision). Primary 06F15; Secondary 08B20, 08A50.

This research was supported in part by NSF Grant MCS 8203615.

(C) 1986 American Mathematical Society $0002-9939 / 86 \$ 1.00+\$ .25$ per page 
of all normal valued $l$-groups and is defined by the condition $x_{1} x_{2} \leq x_{2}^{2} x_{1}^{2}$ for all $x_{1}, x_{2} \geq e$, which may be written as the identity

$(*) \quad\left(x_{1} \vee e\right)\left(x_{2} \vee e\right) \wedge\left(x_{2} \vee e\right)^{2}\left(x_{1} \vee e\right)^{2}=\left(x_{2} \vee e\right)\left(x_{1} \vee e\right)$ for all $x_{1}, x_{2}$

Hence any proper subvariety of $\mathbf{L}$ is a subvariety of $\mathbf{N}$ and satisfies the identity $(*)$.

Thus, an algorithm $M_{2}$ which enumerates all consequences of the union of the axioms of $\mathbf{L}$ and the identity $u=v$ will eventually list the identity (*) if and only if $u=v$ is not a consequence of the axioms of $\mathbf{L}$.

The algorithm for solving the word problem for free $l$-groups is now clear. For any given identity $u=v$, we begin the procedures $M_{1}$ and $M_{2}$. The procedure stops when $u=v$ appears in the list enumerated by $M_{1}$ or when $(*)$ appears in the list enumerated by $M_{2}$.

For the generalization of this to arbitrary algebras, we consider a f.p. variety $\mathbf{V}$ such that there is some effectively given list $\mathbf{V}_{1}, \mathbf{V}_{2}, \mathbf{V}_{3}, \ldots$ of proper subvarieties such that

(i) every proper subvariety of $\mathbf{V}$ is a subvariety of some $\mathbf{V}_{\boldsymbol{i}}$,

(ii) we know, for each $\mathbf{V}_{i}$, an identity $w_{i}=w_{i}^{\prime}$ satisfied by $\mathbf{V}_{i}$ but not by $\mathbf{V}$. Then the word problem is solvable for free $\mathbf{V}$-algebras. We leave the proof to the reader.

Note that this procedure is applicable to $\mathbf{A}$, the variety of all abelian $l$-groups and to the Scrimger varieties [5] covering $\mathbf{A}$. The referee has also pointed out some other applications to varieties of $l$-groups. For each prime $p, L_{p}$ is the variety of all $l$-groups satisfying $x^{p} y^{p}=y^{p} x^{p}$. Scrimger [5] has shown that $L_{m} \subseteq L_{n}$ if and only if $m \mid n$. Gurchenkov [2] has constructed $l$-groups $a(r, p)$ for each prime $p$ and positive integer $r$ and shown that the varieties $S_{p} r$ generated by each of these groups are the only subvarieties of the corresponding $L_{p}$. Since each $S_{p} r$ may be defined by a single identity, it follows that the free $l$-groups in the varieties $L_{p}$ and $S_{p} r$ all have solvable word problem. For $r=1$, we get the Scrimger varieties mentioned above.

\section{REFERENCES}

1. T. Evans, K. Mandelberg and M. F. Neff, Embedding algebras with solvable word problem in simple algebras-some Boone-Higman type theorems, Logic Colloquium '73, Studies in Logic, vol. 80, North-Holland, Amsterdam, 1975, pp. 259-277.

2. S. A. Gurchenkov, Varieties of l-groups with the identity $\left[x^{p}, y^{p}\right]=1$ have finite bases, Algebra and Logic 23 (1984), 27-47.

3. W. C. Holland, The largest proper subvariety of lattice-ordered groups, Proc. Amer. Math. Soc. 57 (1976), 25-28.

4. W. C. Holland and S. H. McCleary, Solvability of the word problem in free lattice-ordered groups, Houston J. Math 5 (1979), 99-105.

5. E. B. Scrimger, A large class of small varieties of lattice-ordered groups, Proc. Amer. Math. Soc 51 (1975), 301-306.

Department of Mathematics, Emory University, Atlanta, Georgia 30322 